\title{
Incremental ECAP as a method to produce ultrafine grained aluminium plates
}

\author{
Malgorzata LEWANDOWSKA ${ }^{1, a^{*}}$, Witold CHROMINSKI ${ }^{1, b}$, \\ Marta LIPINSKA ${ }^{1, \mathrm{c}}$, Lech OLEJNIK ${ }^{2, \mathrm{~d}}$ and Andrzej ROSOCHOWSKI ${ }^{3, \mathrm{e}}$ \\ ${ }^{1}$ Warsaw University of Technology, Faculty of Materials Science and Engineering, Woloska 141, \\ 02-507 Warsaw, Poland \\ ${ }^{2}$ Warsaw University of Technology, Institute of Manufacturing Processes, Narbutta 85, 02-524 \\ Warsaw, Poland \\ ${ }^{3}$ Design, Manufacture and Engineering Management, University of Strathclyde, 75 Montrose \\ Street, Glasgow G1 1XJ, United Kingdom \\ amalew@inmat.pw.edu.pl, bwichr@inmat.pw.edu.pl, 'martal54op@gmail.com, \\ dolejnik@wip.pw.edu.pl, ea.rosochowski@strath.ac.uk
}

Keywords: Incremental ECAP, ultrafine grained materials, aluminium plates.

\begin{abstract}
In this work, we propose a new approach to produce ultrafine grained plates using a modified ECAP method, namely incremental ECAP. Unlike conventional ECAP, incremental ECAP works in small steps in which deformation and feeding are divided between two different tools acting asynchronously. Incremental processing reduces forces and allows processing relatively large billets. The major advantage of this technique is that the specimens are in the form of plates with rectangular shape, which makes them suitable for further processing, e.g. via deep drawing. We report here a study on microstructure development, mechanical properties and their anisotropy in aluminium plates processed by incremental ECAP. Eight passes applied (accumulated strain of 9.2) with rotation about $\mathrm{Z}$ axis brought about the reduction of grain size down to $600 \mathrm{~nm}$ with $80 \%$ fraction of high angle grain boundaries and very homogenous equiaxial microstructure. This resulted in a significant increase in mechanical strength with an ultimate tensile strength reaching $200 \mathrm{MPa}$ and more importantly very low anisotropy with respect to rolling direction.
\end{abstract}

\section{Introduction}

Ultrafine grained (UFG) materials have attracted significant scientific interest due to their superior mechanical properties [1,2]. Equal channel angular pressing (ECAP) is one of the most common processes that allows accumulating very high strains (difficult to obtain by any other conventional deformation technique) and obtaining UFG structure in a number of metals and alloys [3]. One of the major advantages of ECAP processing is the possibility of producing materials that are capable of superplastic elongation at strain rates approximately two orders of magnitude faster than those currently employed for commercial superplastic forming operations [4]. However, superplastic forming operations require the material in the form of thin sheets. Therefore, there is a need to develop an ECAP based technology that allows producing material in such a form.

In this work, we would like to demonstrate the capabilities of a new approach to produce UFG plates using a modified ECAP method, namely incremental ECAP. Unlike conventional ECAP, incremental ECAP works in small steps in which deformation and feeding are divided between two different tools acting asynchronously. Incremental processing reduces forces and allows processing relatively large billets. The major advantage of this technique is that the specimens are in the form of plates with rectangular shape [5,6], which makes them suitable for further processing, e.g. via deep drawing or friction stir welding [7]. The particular attention was paid on microstructural homogeneity and the anisotropy of mechanical properties in correlation to the applied processing route. 


\section{Experimental}

The material studied in this work was technically pure 1050 aluminium. Prior processing it was in the cold rolled state. The plates of dimensions $62 \times 62 \times 3 \mathrm{~mm}^{3}$ were subjected to 8 passes of incremental ECAP using two different routes: (1) with rotation by $180^{\circ}$ about $\mathrm{X}$ axis (extrusion direction) after each pass and (2) with rotation by $90^{\circ}$ about $\mathrm{Z}$ axis (normal to plane) -after each pass. The detailed description of incremental ECAP processing can be found elsewhere [5]. Schematics of I-ECAP used to process of square plates was shown in Fig. 1. Three tools are required for pressing flat billets incrementally through an angular channel. A die, which consists of two parts (1a-stationary and $1 \mathrm{~b}$-movable), a punch 2 and a pusher 3 . After feeding the billet 4 the die set is closed to constitute the input channel. Next reciprocating punch moves down to close the output channel and to shear just fed portion of the square plate. Arrows are used to indicate character of movements for tools, required to fulfil the sequence of incremental pressing through a channel. As one can see in Fig. 1, a half of the billet was already processed in this operation of an equal channel angular pressing with the channel angle of $90^{\circ}$.

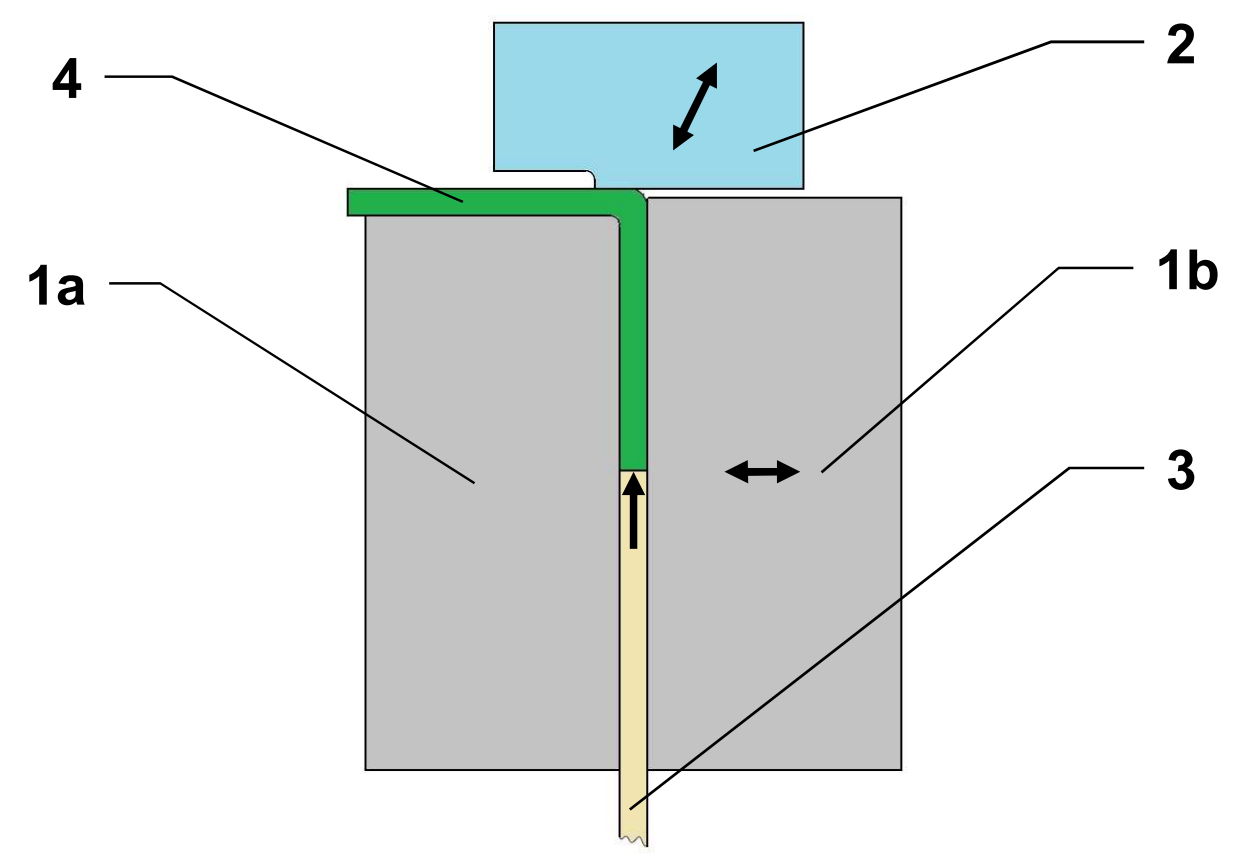

Fig. 1. Schematics of I-ECAP for processing of square plates

In short, it should be noted that in terms of the deformation type and value of the strain induced, the proposed process is equivalent to ECAP, i.e. deformation is by pure shearing and the total equivalent strain in one pass is $\varepsilon=1.15$, which is the value known from the conventional ECAP. Incremental ECAP is carried out in a series of small deformation by pushing the billet forward by a distance of $0.6 \mathrm{~mm}$ and shearing the fed material stepwise.

The microstructure was investigated by means of EBSD orientation mapping performed on Hitachi SU70 analytical scanning electron microscope (acceleration voltage of 20kV) equipped with a Schottky emitter. The data collected during mapping were subsequently used to determine the grain equivalent diameter $\left(\mathrm{d}_{2}\right)$, defined as a diameter of circle with the same area as the measured grain, and distributions of grain boundary misorientation angles.

Microhardness test was performed with standard Vickers indenter under a load of $200 \mathrm{~g}$. Low load value was chosen for higher sensitivity on microstructure heterogeneities. The tests were performed on the cross sections to detect its homogeneity along plate's thickness. In addition, tensile tests were carried out using flat mini samples, as described in [8]. Tensile samples were cut from two directions - perpendicular and longitudinal to the direction of the last pass. Digital Image Correlation (DIC) has been used for accurate strain determination. 


\section{Results and discussion}

\section{Mechanical properties}

Fig. 2 represents a plot of microhardness measurements across plate's thickness starting from the top surface which had a contact with a punch tool (for reference see Fig. 1). It can be seen that for both applied deformation routes microhardness increases with increasing number of passes. However, it should be noted that after the first pass (green line) the microhardness values clearly decrease with distance from the top surface. The difference between these two planes is 5 HV0.2 and is an effect of shear zone heterogeneities which influence local level of strain. Such an effect is typical for the first ECAP pass despite used tool arrangement, as described in details in Ref. [8]. As the applied strain increases, these differences diminishes and microhardness level remains relatively constant, especially for the plates deformed with the rotation about $\mathrm{Z}$ axis. These results clearly indicate that both applied deformation routes lead to uniform mechanical properties on the plates' thickness.
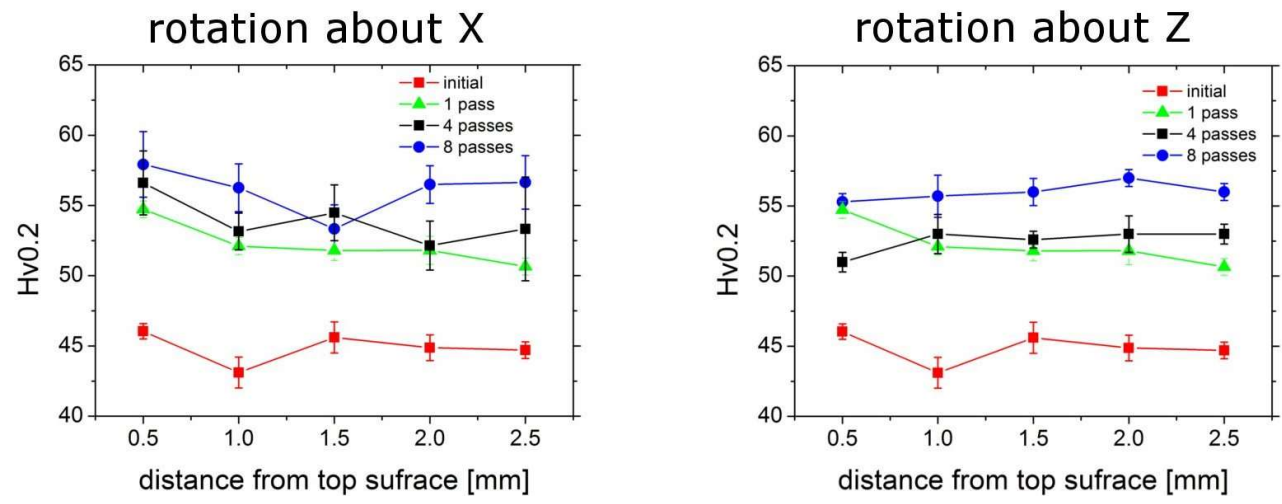

Fig. 2 Microhardness changes measured on $Y$ plane of plates produced with different deformation routes number of passes

To get an insight into the anisotropy of mechanical properties in two perpendicular directions (i.e. extrusion and transverse deformation direction, ED and TD, respectively) a series of tensile tests were performed. The yield strength measured along ED increased from 124 for initial material to 154 and $167 \mathrm{MPa}$ after 8 passes for rotation about $\mathrm{X}$ an $\mathrm{Z}$ axes, respectively. The graph presented in Fig. 3 represents the plot of a ratio of yield strengths measured along ED and TD to quantify the anisotropy of mechanical properties in the plates produced using the two deformation routes. As it can be seen, the plates differ significantly, i.e. the rotation about $Z$ axis leads to a relatively uniform mechanical properties (yet after 4 passes) with the ratio of yield strengths measured along ED and TD close to 1 while the rotation about $\mathrm{X}$ axis leads to a relatively strong anisotropy with yield strength measured along ED being lower than along TD. It should be noted that the first pass causes the strongest anisotropy, which become reduced with increasing I-ECAP passes.

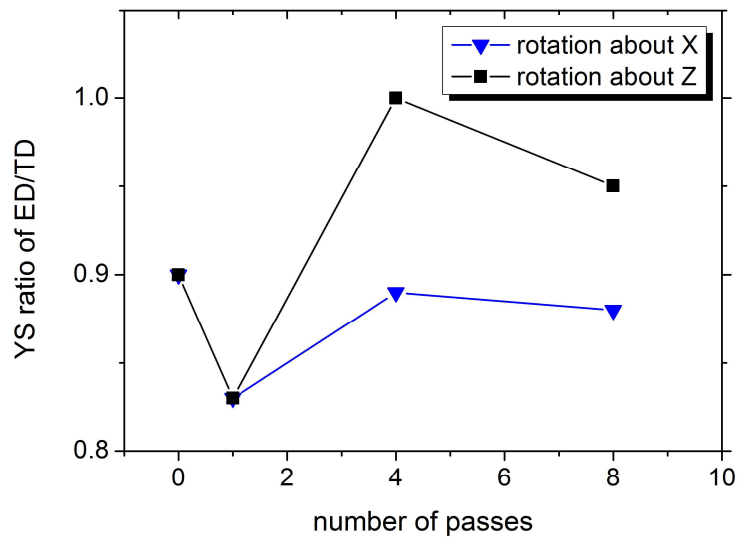

Fig. 3 Ratio of tensile strength measured along deformation direction and perpendicularly to deformation direction on plates produced with different deformation routes with different number of passes 


\section{Microstructure}

To understand the mechanical behavior of 1050 aluminium plates processed by incremental ECAP, a detailed EBSD analysis of three characteristic planes as defined in Ref. [10] were performed. The result of observations of the samples after eight passes for both applied deformation routes are gathered in Fig. 4.

At first glance significant differences for both deformation routes can be noticed. Plates rotated about $\mathrm{X}$ axis feature bands of similar orientation (the same color code) separated by high angle boundaries. However, inside these bands only a network of low angle grain boundaries is present, which is a consequence of the fact that only two shearing planes available during deformation with rotation about $\mathrm{X}$ axis. As a result, only a modest set of dislocation slip systems can be activated and equiaxial grain structure cannot be produced due to limited dislocations activities, as reported in Ref. [11]. With increasing number of passes, primary grains become elongated with deformation direction on $\mathrm{Y}$ and $\mathrm{Z}$ planes and low angle grain boundary substructure within them arises. The final grain size (including subgrains with misorientation angle higher than $3^{\circ}$ ) is $650 \mathrm{~nm}$.

Rotation about $\mathrm{Z}$ axis produces uniform grain size distribution with very fine size on every plane. No elongation related with primary grains, as in the previous samples, can be noticed which leads to conclusion that this route is very efficient in terms of grain refinement. Adjacent grains differ in colors as a result of high misorientation angles between them. Rotation about $\mathrm{Z}$ axis every subsequent pass activate different set of slip systems which reoccur every four passes - two times per full applied deformation procedure. Then more equiaxial structure can be produced than in rotation about $\mathrm{X}$ axis and second rotation (fifth to eighth passes) lead to increase of misorientation angle between already produced subgrains. This leads to smaller grain size with the mean value of $580 \mathrm{~nm}$ (including subgrains with misorientation angle higher than $3^{\circ}$ ).
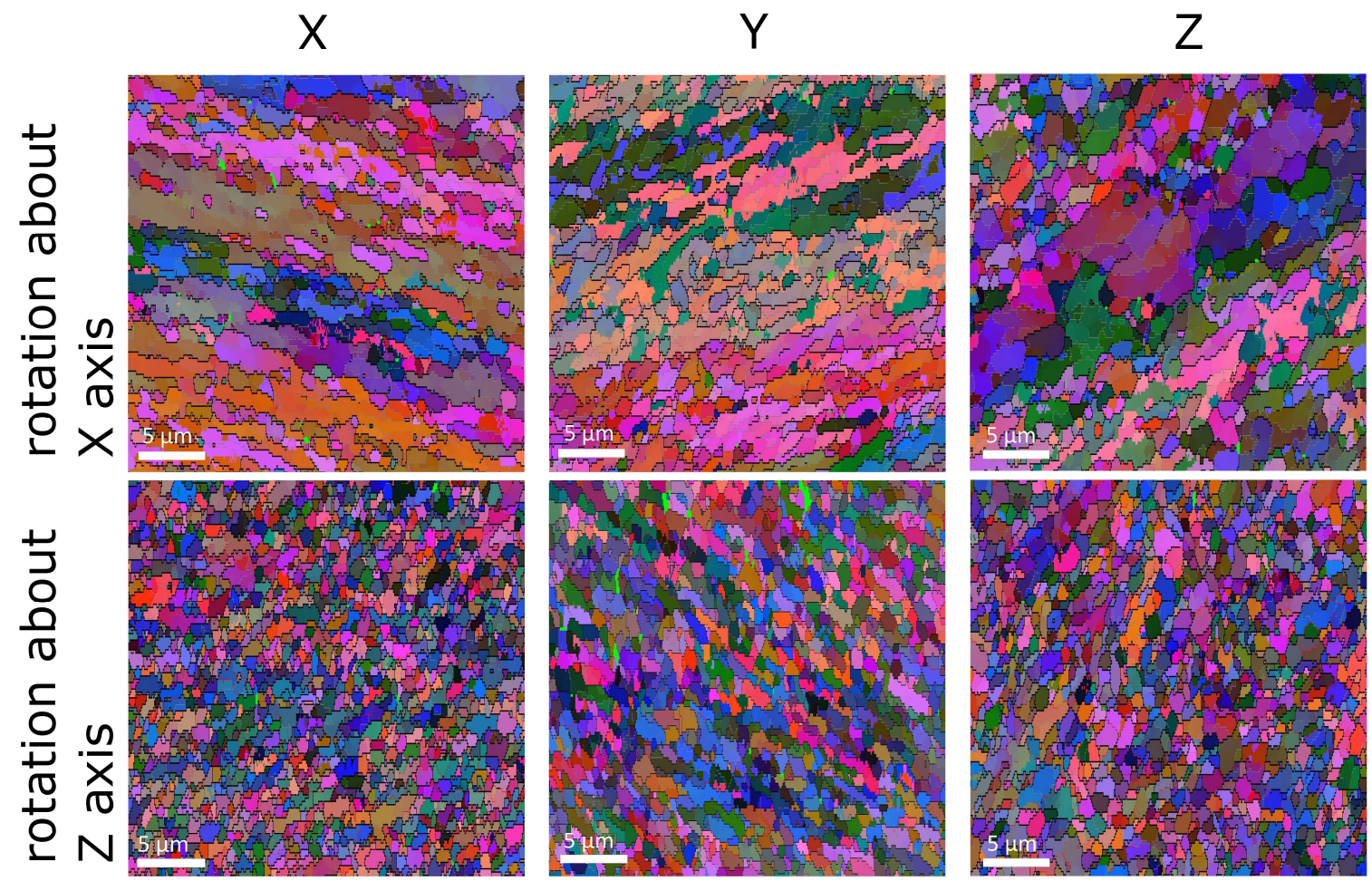

Fig. 4 Comparison of EBSD orientation maps obtained on three different planes in plates deformed with rotation about $X$ and $Z$ axes

Changes in misorientation angle distributions can be followed in Fig. 5. It can be seen that rotation about $\mathrm{Z}$ axis is much more efficient in terms of grain rotations during subsequent I-ECAP passes as after eight passes misorientation angle distribution approaches random. After the final pass, the content of high angle grain boundaries is 58 and $81 \%$ for rotation about $\mathrm{X}$ and $\mathrm{Z}$ axes, respectively. 

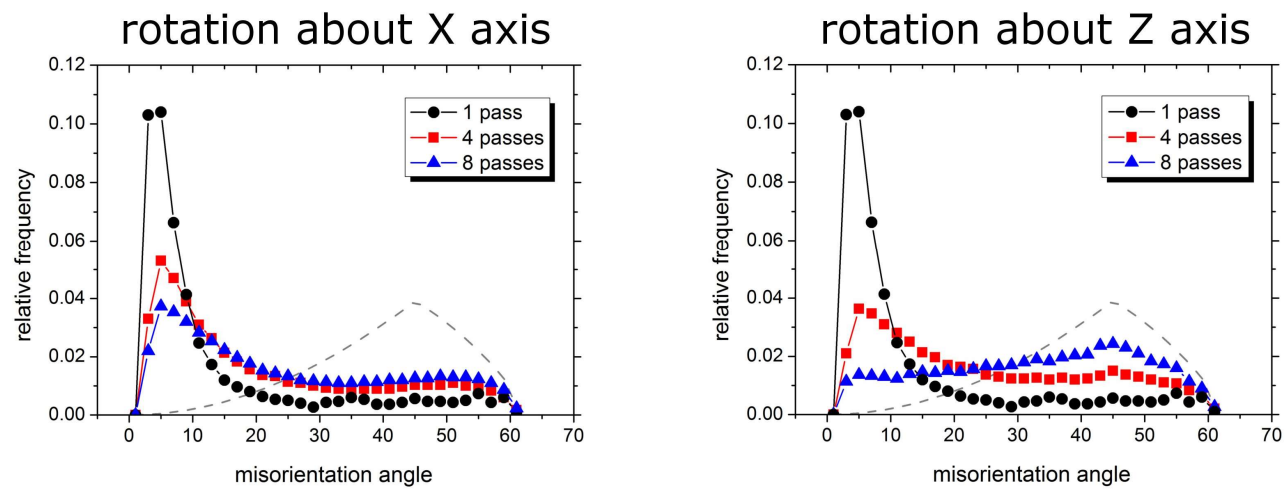

Fig. 5 Misorientation angle distributions for grain boundaries in plates deformed with rotation about $\mathrm{X}$ and $\mathrm{Z}$ axes, dashed line is a MacKenzie plot for random distribution

Differences of general microstructure appearance are the key to understand the anisotropy of mechanical properties in investigated samples. Rotation about $\mathrm{X}$ axis produces lamellar type structure aligned with deformation direction. When a material is tested along ED, these lamellas are stretched and the average distance between high angle grain boundaries is much larger than in the case of testing along TD. Then according to Hall-Petch relation which indicates grain boundaries as obstacles in dislocation motion it can be stated that material's resistance to plastic deformation is much higher when lamellas are perpendicular to the tensile direction. When the microstructure becomes more homogenous (as in samples deformed with rotation about $\mathrm{Z}$ axis) no lamellas can be distinguished and no preferred directions. The grains are equiaxial and significant amount of high angle grain boundaries provides many obstacles to mobile dislocations. As no alignment with ED is observed, mechanical properties are independent on testing direction. Thus, the rotation about $\mathrm{Z}$ axis with subsequent I-ECAP passes produce a material with homogenous microstructure and mechanical properties.

\section{Summary}

$>$ It has been demonstrated that incremental ECAP is an efficient way to produce UFG plates.

$>$ Two routes have been tested, i.e. with rotation about $\mathrm{X}$ and $\mathrm{Z}$ axis, with the former being much more effective in terms of equiaxial microstructure and HAGBs formation.

$>$ Eight passes with rotation about $\mathrm{Z}$ axis resulted in grain size reduction to below $600 \mathrm{~nm}$ and very high fraction of high angle grain boundaries of about $80 \%$ while rotation about $\mathrm{X}$ axis results in grain size below $700 \mathrm{~nm}$ with relatively low fraction of high angle grain boundaries $(58 \%)$.

$>$ The plates have reasonably isotropic mechanical properties, which make them suitable for further processing.

Acknowledgement. This research was financed by the National Centre for Research and Development within the project "Properties and longterm behaviour of dissimilar joints for aerospace applications" (Contract no. WPN/6/2013).

\section{References}

[1] R. Valiev, Y. Estrin, Z. Horita, T.G. Langdon, M.J. Zehetbauer, Y.T. Zhu, Producing Bulk Ultrafine-Grained Materials by Severe Plastic Deformation, JOM. (2006) 33-39.

[2] R.Z. Valiev, Y. Estrin, Z. Horita, T.G. Langdon, M.J. Zehetbauer, Producing Bulk UltrafineGrained Materials by Severe Plastic Deformation : Ten Years Later, JOM 68 (2016) 12161226. doi:10.1007/s11837-016-1820-6. 
[3] R.. Valiev, R.. Islamgaliev, I.. Alexandrov, Bulk nanostructured materials from severe plastic deformation, Prog. Mater. Sci. 45 (2000) 103-189. doi:10.1016/S0079-6425(99)00007-9.

[4] R.Z. Valiev, T.G. Langdon, Principles of equal-channel angular pressing as a processing tool for grain refinement, Prog. Mater. Sci. 51 (2006) 881-981. doi:10.1016/j.pmatsci.2006.02.003.

[5] W. Chrominski, L. Olejnik, A. Rosochowski, M. Lewandowska, Grain refinement in technically pure aluminium plates using incremental ECAP processing, Mater. Sci. Eng. A. 636 (2015) 172-180. doi:10.1016/j.msea.2015.03.098.

[6] L. Olejnik, A. Rosochowski, M. Richert, Incremental ECAP of plates, Mater. Sci. Forum. 584-586 (2008) 108-113.

[7] M. Lipińska, L. Olejnik, A. Pietras, A. Rosochowski, P. Bazarnik, J. Goliński, et al., Microstructure and mechanical properties of friction stir welded joints made from ultrafine grained aluminium 1050, Mater. Des. 88 (2015) 22-31. doi:10.1016/j.matdes.2015.08.129.

[8] O.J. Weiß, E. Gaganidze, J. Aktaa, Materials Challenges and Testing for Supply of Energy and Resources, 1 (2012) 221-234. doi:10.1007/978-3-642-23348-7.

[9] C. Xu, M. Furukawa, Z. Horita, T.G. Langdon, The evolution of homogeneity and grain refinement during equal-channel angular pressing: A model for grain refinement in ECAP, Mater. Sci. Eng. A. 398 (2005) 66-76. doi:10.1016/j.msea.2005.03.083.

[10] Y. Iwahashi, Z. Horita, M. Nemoto, T.G. Langdon, The process of grain refinement in equalchannel angular pressing, Acta Mater. 46 (1998) 3317-3331. doi:10.1016/S13596454(97)00494-1.

[11] A. Gholinia, P.B. Prangnell, M. V. Markushev, Effect of strain path on the development of deformation structures in severely deformed aluminium alloys processed by ECAE, Acta Mater. 48 (2000) 1115-1130. doi:10.1016/S1359-6454(99)00388-2. 